\title{
Impact of early comprehensive geriatric assessment in the acute medical unit
}

\author{
Authors: Charlotte Owen and Divya Tiwari
}

\section{Aims}

Older people presenting to the acute medical unit (AMU) in our hospital were traditionally triaged to acute medicine (ACM), discharged after shorter stay ( $<72$ hours) or medicine for the elderly (MFE). There is evidence that comprehensive geriatric assessment in AMU improves functional and cognitive outcomes in older adults.

\section{Methods}

A new triage of elderly acute medicine (eACM) now provides day-to-day care and early geriatric multidisciplinary assessment through a team composed of a consultant geriatrician, specialist nurses and therapists. After discharge, patients are often followed up in a rapid assessment clinic. There were 5,857 admissions to the AMU from 1 May 2013 to 30 September 2013, of which 2,371 were older than 80 years. Out of these, 553 were triaged eACM and 621 were triaged MFE. We conducted casenotes review for 50 out of 553 patients.

\section{Results}

Median age was 86 (72-101) and 74\% lived in their own home, $76 \%$ of whom lived independently. $20 \%$ of patients presented with a fall, $70 \%$ of which were attributed to non-physical causes. The most common diagnosis made was pneumonia/LRTI (18\%).

$82 \%$ were assessed by a consultant geriatrician within 24 hours; $91 \%$ of those requiring further assessment were reassessed within the subsequent 24 hours. Therapy review was conducted in 50\% within 24 hours. Median LOS was 6.5 days, $52 \%$ LOS $\leq 72$ hours and $36 \%$ were re-triaged to MFE. There were no clear predictors for prolonged LOS. $25 \%$ of discharges from eACM were with an increased level of social support.

\section{Conclusions}

eACM triage rates were variable among clinicians; however, this model was successful in bringing early comprehensive geriatric assessment of older patients admitted to AMU. Most patients were assessed by a consultant geriatrician within 24 hours of admission. Although therapy assessment was often delayed, the team discharged $52 \%$ of patients within 72 hours, $25 \%$ of patients with increased social support.

\section{Conflict of interest statement}

No conflicts of interest to declare. 